\title{
The Astronomical Data Warehouse
}

\author{
Clive G. Page
}

Department of Physics \& Astronomy, University of Leicester, Leicester, LE1 7RH, UK

\begin{abstract}
The idea of the astronomical data warehouse has arisen as the natural extension of current trends in astronomical data archives, and from an analysis of the types of query likely to be sent to a virtual observatory. Data warehouses will be centres providing both data and computational facilities.
\end{abstract}

\section{Querying the Virtual Observatory}

Members of the AstroGrid project have some background in the provision of data archive services and of using them, but in designing the virtual observatory we have been driven principally by some recent collections of typical astronomical problems and use-cases:

- The set of some 47 science problems set out on the Astrogrid Wiki pages[1]. - The collection of (so far) 29 use-cases assembled by the US-NVO Project[2].

- A set of 35 queries for the Sloan Digital Sky Survey[3].

Most of these involve a series of interactions with the virtual observatory, which can be further broken down into more basic data access operations. These can be roughly classified as follows: firstly positional queries, second observational queries, and thirdly all other types (which will be called 'non-positional' for convenience).

\subsection{Positional Queries}

Positional queries are ones seeking information about a specific small patch of sky, or on a named object. An object name can usually be translated to a position using a name resolver such as Simbad or NED. Given the errors in coordinates, in either case the query is actually one about a small area of sky, typically a circular region about a given (RA, DEC): this has become known as the cone search. Typical examples might be:

- Are there any X-ray sources near HD123456?

- Can I have all infra-red images centred on PSR0123-456?

Because of their importance in astronomy, positional queries make up a high proportion of all queries to on-line resources, but there may be some bias caused 
by the fact that many existing archives specialise in the cone search, and some support little else.

It now seems generally accepted that one of the prime requirements of the $\mathrm{VO}$ is some sort of Resource Registry, which will direct user's queries to the sites most likely to provide them with relevant information. Pilot projects to implement the cone search using modern XML-based protocols such as SOAP

and WSDL, and to standardise the results using the VOTable format, are under way (as noted by other papers in these proceedings).

\subsection{Observational Queries}

Much useful on-line data arises not from surveys but from specific observations of the sky. Those with sufficient expertise can locate observations which may have covered their objects of interest and download the raw or semi-processed data to their own computer for local re-analysis. Here are two examples of such queries:

- Can I download the HST data from all observations of NGC9876?

- Are the XMM-Newton data from observing HD123456 made public yet?

Such queries are currently limited to those who have expertise in the relevant branch of astronomy, the appropriate software, and enough computing power necessary to make good use of the resulting data. With the advent of the $\mathrm{VO}$, and with more observatories running standard data reduction pipelines, such downloads may decline in importance. Even experts, however, are likely to benefit from the resource discovery mechanisms being proposed for the VO, and from the security architecture, which should provide a uniform mechanism for access to both public and private data.

\subsection{Non-positional Queries}

This category is extremely diverse, since it includes everything not covered in the other two. Examples include:

- Cross-matching objects in catalogues from different wavebands: the basis of many types of more detailed investigation.

- Simple selections on ranges of parameters, such as selecting on (B-V) or X-ray/Optical flux ratio.

- Statistical investigations such as finding rare objects as outliers, and regression analysis to find trends and correlations.

- Clustering algorithms, classification algorithms, and measurements of spatial correlation functions.

- Visualisations and other methods of using the human eye to aid pattern discovery.

- Time-series operations: finding and classifying variability, using power-spectra to find periodicities, etc.

- Discovering moving objects by comparing images or positional measurements taken over a period of time. 


\section{Handling Non-positional Queries}

Many of the operations identified above will be I/O-intensive, or cpu-intensive, or both. Some of them can be carried out using a relational database management system (DBMS), but many others (time-series analysis, clustering algorithms, image processing) will require specialised software. This may be available from existing packages (AIPS, CIAO, FTOOLS, IRAF, MIDAS, Starlink, XMM-SAS, etc.) or may require the development of new algorithms and software.

To get VO up and running quickly we should concentrate initially on operations that can be done well using existing software, such as those supported by a relational DBMS, which is likely to be installed at almost every data archive site. A notable feature of the DBMS is the ability to create and use indices.

As tabular datasets increase in size the efficiency gain from indexing also grows, almost linearly. Suppose we try to find a given row in a table such as the USNO-A2 catalogue, which has 626 million rows: if a suitable index has been created this should require only two or three disc seeks, taking only 20 or 30 ms. But even the simplest operation which requires trawling through the entire table will take several hundred seconds. So the ratio in this case is of the order of 10,000:1.

Unfortunately not all operations can be speeded up with indices. Many tables have simply too many columns for this to be practical: several tables in the Vizier collection [4], for example, have over 100 columns, most of them fields which a user might want to search. In addition some users will want to select on say the difference between two magnitude columns (or ratio of two fluxes): here indices created on individual columns do not help. There are also operations which cannot avoid a complete sequential scan, for example some statistical queries, and of course index creation itself. Most join operations (and all outer joins) need to access every row in one of the two tables involved.

Many existing data archive sites limit external users to operations which make no more than modest use of resources (for example the cone-search with an index on celestial position); a few have a more liberal policy, but of course external users take their place in the queue with everyone else, and there is the ever-growing risk of abuse of such open facilities.

As noted earlier, scientifically valuable results often arise from combining data from two (or more) different sites: this usually involves making substantial data transfers across the wide area network. Although network capacities are continually rising, with many international links running at $155 \mathrm{Mb} / \mathrm{s}$ or even higher, the effective bandwidth for file transfers seems considerably slower in practice. The particle physics community[5], whose networking needs are even greater than ours, have found in hard to get file transfers running internationally at more than $100 \mathrm{Mb} / \mathrm{s}$, and that typical rates on most routes are around 20 $\mathrm{Mb} / \mathrm{s}$, i.e. around one terabyte/week.

For many DBMS operations, low latency is also vital, so the speed of light presents a serious obstacle to performing some database operations over a widearea network. 
All these considerations lead to the conclusion that while the simpler types of query, such as the cone-search, can be carried out on remote servers, many of the more complex ones will need the data to be located close to the processing power - but not necessarily close to the user. In cases where adequate processing power is not available attached to the discs, or not available to external users of a site, then the best alternative is to obtain a copy of the relevant dataset and install it on a more powerful computer facility.

\section{The Data Warehouse Concept}

The term "Data Warehouse" has been used in commerce for some time: in large organisations it is often impractical to carry out complex querying over a large and disparate collection of DBMS, especially if these are spread over a number of physical sites, or are continually being updated through new transactions. The idea of the data warehouse is to set up a facility with a copy of all the important datasets from around the organisation, and get them all in the same format, and indexed optimally.

Since many of the same considerations apply to the world's collection of astronomical data, the term Astronomical Data Warehouse seems reasonably appropriate. What is proposed is a facility which contains:

- A collection of the most popular standard datasets permanently installed;

- Ample spare disc space so that users can download and install such other datasets as they require;

- Adequate processor power, probably though the use of Beowulf or other cluster systems;

- A relational database management system and the usual astronomical packages pre-installed;

- Good network connections to the outside world.

Although there is no reason why data warehouses should not be set up as separate facilities, it seems to me that they will probably be set up as a natural extension of the facilities already provided by the major data centres.

\section{Towards the Data Warehouse}

A trend is already apparent towards moving the data to where processing power is located. Many important datasets and data collections are already present at two or more locations around the world. The HST archive is, of course, mirrored also in Garching; similarly the Chandra data archive has a mirror at LEDAS (Leicester), while the NASA Astrophysics Data System (ADS) also has a mirror in the UK at Nottingham. The popularity of the Vizier collection of catalogues and other tabular data, produced by the Centre de Donées Astronomiques de Strasbourg, can be seen from the fact that it now has eight mirror sites. Some important source catalogues (such as the GSC and USNO-A2 catalogues) and 
images collections (such as the Digital Sky Survey) are already distributed on CD-ROM and installed on disc at many sites. It will soon be exceptional for an important data resource to be located at only one site.

One caveat must be noted: astronomical datasets are produced by complex instruments which need to be fully understood by anyone carrying out the basic data reduction stages and making the results available to external users. The existence of mirror sites does not at all lessen the case for having as curators of the original datasets the astronomers who produced them.

\section{Software for the Data Warehouse}

The software to be installed at an astronomical data warehouse will probably include all of the following:

- A suitable database management system.

- Standard astronomical software packages.

- Statistical packages: there seem to be a many similar packages around, but almost all depend on being able to read the dataset of interest into physical memory. Since many astronomical datasets are already larger than feasible memory sizes, this presents a problem. A good review of available packages is given by by Wegman et al[6].

- Web Services interfaces using SOAP and WSDL.

- Grid Services based on the Open Grid Services Architecture[7], so virtual observatory users can take advantage of computational and data grid facilities.

The AstroGrid project is conducting an evaluation of a number of commercial and freeware relational DBMS: these investigations of DBMS are still incomplete, but a summary of the findings so far is available on-line[8]. One of the prime application areas is likely to be access to catalogues of celestial objects, in which efficient indexing on celestial position (RA,DEC) is essential, especially when performing a fuzzy join between one catalogue and another. It has been assumed that to to this efficiently we needed a DBMS with spatial indexing capability, such as the R-tree indexing in Postgres. Recently a new algorithm has been developed and tested which makes use of an equal-area pixelisation of the sky such as those of HTM[9] or HEALPix[10], though currently the latter is used. This PCODE[11] method seems to be significantly faster than the use of an Rtree index, and it can use any DBMS since uses an equi-join of integers, and has no need for specialised spatial indexing.

\section{Potential Problems}

Many problems remain to be solved, of course, for example

- Will data curators be happy to allow remote data warehouses to take copies of complete datasets? 
- How can large datasets be copied from one site to another? If current network links are inadequate, we need to find alternatives. The VISTA project[12] plans to ship data on physical discs from Chile to Europe when it goes on-line around 2006, and the SDSS project, having similar problems, has proposed terascale sneaker-net[13] as a solution.

- How can we interface DBMS with other scientific packages? This is especially difficult because few systems can import or export more than simple text files, and have practically no support for metadata. Perhaps the adoption of XML-based data formats such as VOTable will help.

- How can astronomers write their own software to interface to large databases?

\section{Acknowledgements}

I would like to acknowledge many valuable discussions with my colleagues in the AstroGrid, AVO, and US-NVO projects, without whom these ideas would have been even less than half-baked.

\section{References}

1. 'AstroGrid science problem list', http://wiki.astrogrid.org/bin/view/Vo/ScienceProblemList

2. US-NVO Project, ' 100 science problems for the NVO', http://monet. astro.uiuc.edu/ rplante/Vo/metadata/100questions.html

3. J. Gray, D. Slutz, A. Szalay, A. Thakar, J. Vandenberg, P. Kunszt, C. Stoughton, 'Data Mining the SDSS SkyServer Database', MSR-TR-2002-01 (2002), http://research.microsoft.com/ grey

4. 'VizieR Catalogue Service', http://vizier.u-strasbg.fr/

5. Personal communication from David Newbold of Bristol University.

6. E. J. Wegman, D. B. Carr, R. D. King, J. J. Miller, W. L. Poston, J. L. Solka, and J. Wallin, 'Statistical Software, Siftware and Astronomy', http://www.galaxy.gmu.edu/papers/astr.html

7. 'Open Grid Services Architecture', http://www.globus.org/ogsa/

8. 'Astrogrid DBMS Evaluations', http://wiki.astrogrid.org/bin/view/Astrogrid/DbmsEvaluations

9. 'Hierarchical Triangular Mesh (HTM)', http://taltos.pha.jhu.edu/htm/

10. 'Hierarchical Equal Area isoLatitude Pixelisation (HEALPix)', http://www. eso.org/science/healpix/

11. C. Page, 'Indexing the Sky', http://wiki.astrogrid.org/bin/view/Astrogrid/SkyIndexing

12. 'Visible \& Infrared Survey Telescope for Astronomy', http://www.vista.ac.uk/

13. J. Gray, W. Chong, T. Barclay, A. Szalay, and J. Vandenburg, 'TeraScale SneakerNet', MS-TR-02-54 (2002), http://research.microsoft.com/ grey 